\title{
Potential Modulation of Equilibrium and Excitation Phenomena at the Electrolyte-Solid Interface
}

\author{
Progress Report
}

for the period July 1, 1990 - Sept. 30, 1991

by

T. E. Furtak

Physics Department

Colorado School of Mines

Golden, CO 80401-1887

Prepared for

THE U. S. DEPARTMENT OF ENERGY

AGREEMENT NO. DE-FG02-86ER45253

October 1991

This report was prepared as an account of work sponsored by the United States Government. Neither the United States nor the Department of Energy, nor any of their employees, nor any of their contractors, subcontractors, or their employees, makes any warranty, express or implied, or assumes any legal liability of responsibility for the accuracy, completeness, or usefulness of any information, apparatus, product or process disclosed or represents that its use would not infringe privately-owned rights.

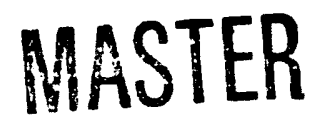




\section{Index}

$\begin{array}{ll}\text { Abstract } & \mathbf{3}\end{array}$

Bibliography of Publications, Reports and Presentations 5

$\begin{array}{ll}\text { Summary of Published Work } & 7\end{array}$

$\begin{array}{ll}\text { Summary of Progress on Other Projects } & 10\end{array}$

Statement of PI and Post Doctoral Involvement 15

$\begin{array}{ll}\text { Statement of Student Involvement } & 16\end{array}$

$\begin{array}{lr}\text { Reprints and Preprints } & 17\end{array}$ 


\begin{abstract}
This progress report covers the work accomplished from the beginning of Sept. 1990, through Sept. 1991. This includes the final portion of the last funding period as well as the first 7 months of the new period.

During this time we have pursued our goal of studying the role of the interfacial potential difference in determining the ground and excited electronic states of the electrolyte-solid system. Ours is a program designed to provide fundamental information about this complicated environment. We seek to improve the techniques with which electrochemical interfaces can be studied and thereby observe details which have never before been measured. We are primarily concerned with optical techniques, but have also integrated conventional and non-conventional supplementary methods.

We have developed the technique of optical second harmonic generation into a spectroscopy by performing the experiment with a wide range of nearly continuous incident photon energies. Through this advantage we have performed detailed tests of the predictions of the electrodynamics of metal surfaces under the perturbation of the interfacial potential as derived through local density functional methods in the jellium limit. Our data show that the theory is reasonably satisfactory when the surface is electron deficient. However, when the surface is negatively charged (electron excess) significant discrepancies are evident. At least part of this deviation has been explained by transitions involving potential modulated surface states. This project has helped clarify some important questions concerning the nonlinear optical properties of metals, as well as lay the groundwork for future applications aimed at monitoring adsorption phenomena with optical second harmonic reflectance spectroscopy.

In a separate application of nonlinear optical measurements on $\operatorname{Ag}(111)$ electrodes we have concentrated on the anisotropic component of the second harmonic reflectance spectrum. We discovered a structural phase transition involving either the first layer of solution material, or the top layer of the $\mathrm{Ag}$ itself. This was characterized by an unambiguous change of symmetry at the transition which is directly observed through the crystallographic orientation dependence of the signal. This surprising result is the only documented restructuring of $\mathrm{Ag}(111)$ in the absence of strongly adsorbed material. It is a good example of the type of new information which can be obtained through complete understanding of a new measurement technique.

Preliminary data covering the second harmonic reflectance spectrum on $\mathrm{Ag}(100)$ have been acquired. This surface has a simple symmetry which allows independent study of the higher multipole effects coming from the bulk. Contrary to the predictions of simple phenomenological models we have detected a potential dependent effect associated with either the bulk of the metal or higher multipole effects in the surface. Either of these explanations will require an evaluation of nonlinear response theory which goes beyond
\end{abstract}


anything which is now available.

We hav i ad a long standing interest in foreign metal monolayers as an example of well-defined strong adsorption in the electrochemical environment. Recently we have applied the quartz crystal microbalance to the $\mathrm{Cu}$ on $\mathrm{Pt}$ system. Our results confirm the simple interpretation that each $\mathrm{Cu}$ atom requires exactly two electrons from the $\mathrm{Pt}$ in the formation of an adsorbed monolayer. This helps resolve a controversy that had developed on the basis of as yet unpublished data from Texas A \& M, taken in a UHV transfer experiment, in which significant charge flow discrepancies were observed.

Our high sensitivity surface Raman facility is nearly operational. Preliminary testing has been encouraging. We have also made good progress on finishing our spectroscopic ellipsometer.

We are pleased with the first phase of our new effort. We feel that this project is exactly on track and will be able to show continued success during the next two stages. 


\section{Bibliography of Publications, Reports, and Presentations}

\section{Publications:}

1. "Direct Comparison of the Chemical Properties of Single Crystal $\mathrm{Ag}(111)$ and Electrochemically Roughened Ag as Substrates for Surface Raman Scattering", S. Byahut and T. E. Furtak, Langmuir, 7, 508 (1991).

2. "Surface Plasmon Assisted Raman Scattering from Electrochemically Controlled Adsorbates on Flat, Single Crystal Silver", S. P. Byahut and T. E. Furtak, Electrochimica Acta, (in press).

3. "Study of Underpotential Deposition of Metals Using the Quartz Crystal Microbalance", Yeke Tang and T. E. Furtak, Electrochimica Acta, (in press).

4. "Optical Second Harmonic Electroreflectance from $\operatorname{Ag}(111) "$, T. E. Furtak, Yeke Tang, and L. J. Simpson, Physical Review B, (submitted).

5. "Ag(111) Surface Reconstruction Studied by Optical Second Harmonic Generation", Yeke Tang, L. J. Simpson, and T. E. Furtak, Physical Review Letters, (submitted).

Invited Presentation:

6. "Surface Plasmon Assisted Raman Scattering from Single Crystal Silver", Federation of Analytical Chemistry and Spectroscopy Societies; Annual Meeting, Cleveland, Oct. 7, 1990, T. E. Furtak and S. Byahut.

\section{Contributed Presentations:}

7. "Second Harmonic Electroreflectance", Interdiciplinary Conference on Electrified Interfaces, Asilomar CA, Sept. 9, 1990. L. Simpson, Yeke Tang, and T. E. Furtak.

8. "Surface Plasmon Assisted Raman Scattering from Electrochemically Controlled Adsorbates on Flat Single Crystal Silver", Interdiciplinary Conference on Electrified Interfaces, Asilomar CA, Sept. 10, 1990. S. P. Byahut and T. E. Furtak.

9. "Anomalies in Underpotential Deposition Revealed with the Quartz Crystal Nanobalance", Interdiciplinary Conference on Electrified Interfaces, Asilomar CA, Sept. 10, 1990. Yeke Tang and T. E. Furtak. 
page 6

10. "Surface Charge Perturbation of the Incident Energy Dependence of Optical Second Harmonic Generation", American Physical Society, March Meeting, Cincinnati OH, March 21, 1991. Yeke Tang, L. J. Simpson, and T. E. Furtak.

11. "Optical Second Harmonic Reflectance Spectroscopy from Metal Electrodes", DOE Corrosion Contractors Meeting, Brookhaven National Laboratory, Sept. 19, 1991. T. E. Furtak, Yeke Tang, and L. J. Simpson. 


\section{Summary of Published Work}

Second Harmonic Electroreflectance Spectroscopy [Bibliography items 4, 5, 7, 10,11]

In our most important development we have discovered a structural phase transition at the interface between $\mathrm{Ag}(111)$ and an aqueous sulfate electrolyte which is driven by changes in the interfacial potential. The significance of this is that the (111) face of fcc metals is normally stable. Only $\mathrm{Au}$ and recently $\mathrm{Pt}$, among all the elements, demonstrate any kind of surface reconstruction for this orientation. Our results show that, at least in the presence of adsorbed $\mathrm{SO}_{4}{ }^{2-}$, there is an instability for $\mathrm{Ag}$ as well. We feel that this transformation may be similar to what has been observed on $\mathrm{Ag}(111)$ in UHV in the presence of adsorbed iodine. There, $30 \%$ of the top $\mathrm{Ag}$ atoms change their height with respect to the bulk so as to create a $\sqrt{3} \times \sqrt{3}\left(R 30^{\circ}\right)$ structure. This, by itself has $C_{3 v}$ symmetry, however the mirror plane of the reconstructed layer is rotated by $90^{\circ}$ with respect to the bulk lattice. Since optical SHG monitors more than one layer of the sample, we are sensitive to, not only the top layer, but also to the first few layers beneath it. Since these have $C_{3 v}$ symmetry as well with the rotated mirror plane, the overall result of the reconstruction is to create a surface whose total symmetry is $C_{3}$. The differences between $C_{3 v}$ and $C_{3}$ are unambiguous in the experimental data. We have examined the approach to $\mathrm{C}_{3}$ behavior as a function of the interfacial potential and the incident photon energy. We find that our ability to detect the transition is restricted to a narrow energy range which, in the second harmonic, is centered around $3.8 \mathrm{eV}$. This indicates that a resonant phenomenon, associated with the transformed surface, is at work. Since the resonance occurs precisely where we expect the optical properties of $\mathrm{Ag}$ to have strong dependencies on the harmonic photon energy, we have concluded that the transformation is in the Ag, not in the adjacent electrolyte. Upon further study we find that, at constant photon energy, the approach to the transition as a function of pntential displays a power law relation when expressed in terms of the relative $\mathrm{C} 3 \mathrm{v}$ signal. The critical exponent for the power law is near 0.4 , similar to what has been seen in second order phase transitions in many divers physical phenomena. Criticality in a new physical system are always of wide interest in the physics community. Current models for surface reconstruction in the fcc metals are based upon strain relaxation in total energy calculations including relativistic effects. The heavier elements are thought to reconstruct because of the influence of the relativistic corrections. It is clear that, in the presence of an adsorbed species like the sulfate in our case, the energy balance at the surface is very subtle and can be easily disrupted.

We can separate the portion of the second harmonic reflectance which depends on the "free electron" properties of the metal by extracting the isotropic component of the signal. We have studied this as a function of the applied potential, which alters the ground state 
charge distribution, and as a function of the incident photon energy. Our object was to provide a quantitative test of the most sophisticated time dependent local density functional theory for metal electrodynamics in the nonlinear response regime. Prior to our work, a group in France had tested the model using an electrochemically perturbed $\mathrm{Ag}(111)$ sample, however their experiment was only performed at one incident photon energy, at $1.17 \mathrm{eV}$. We have covered the entire range of important elementary excitations in $\mathrm{Ag}$ with our results. To avoid the problems associated with normalization from energy to energy, we expressed our data in terms of the zero charge condition of the sample. This sample-normalized differential reflectance spectrum depends only upon the linear optical properties of bulk $\mathrm{Ag}$ (which we obtain from handbook data), and the nonlinear source terms which we have extracted from the local density functional theory. Our results show that the theory works much better than expected when $\Delta \mathrm{Q}>0$, provided the region around the interband transitions are avoided. Since the theory was contrived in the jellium approximation, which does not allow for band structure effects, this is not unexpected. The correlation is much worse when $\Delta \mathrm{Q}<0$. We were unable to get the theory to fit our data in any part of the spectral range, even when the nonlinear source terms were adjusted by a scale factor. Part of the discrepancy is due to surface state transitions which we have identified under these conditions. There is a crystal potential derived surface state on $\mathrm{Ag}(111)$ which lies just above the Fermi level on the uncharged surface. When $\Delta Q$ is made negative, this state moves below the Fermi level, thus becoming a legitimate initial state for a transition to the image potential derived surface state at about $3.4 \mathrm{eV}$ above the Fermi level.

This set of experiments represents the most detailed analysis of the nonlinear optical properties of a metal surface that have been performed to date. We are continuing this effort so as to establish a body of reference data from which other interfacial surface properties of $\mathrm{Ag}$ can be studied using nonlinear optical effects.

\section{Surface Plasmon-Polariton Assisted Raman Scattering. [Bibliography Items 1, 2, 6, 8]}

We obtained the world's strongest Raman signal from a single layer of molecules adsorbed on a flat, single crystal surface. This was accomplished using our unique "Kretchmann configuration" surface plasmon coupling device and multichannel detection. The apparatus has been described in previous Progress Reports. It exploits the large surface optical fields which are associated with surface plasmon-polariton waves on Ag. The Raman effect is driven at the surface by the very large fields. In the second step of the scattering, the emission of the energy shifted radiation, we also couple to surface plasmon-polaritons. This double coupling process results in very high efficiency for getting the optical energy into and out of the monolayer. The Kretchmann method requires that the sample be illuminated from the back side of the metal, which must therefore be a thin film. We grew our Ag epitaxially on mica to create an atomically flat collection of single crystals. This flake of mica was then optically contacted to the back 
side of a hemispherical prism. The Raman shifted light comes out in a hollow cone which we collect in its entirety with a paraboloid-shaped mirror. The spectral analysis was performed with a triple spectrometer coupled to a super-cooled linear photodiode array detector. We used para-nitrosodimethylaniline (pNDMA) as the test molecule, studying it under a variety of conditions at an air interface and finally with the sample contacting the solution of an electrochemical cell. Our strongest signal showed a S/N ration in excess of 400 with this system. We verified that the adsorption of pNDMA led to reversible behavior as a function of the applied potential, unlike the common situation with surface enhanced Raman scattering (SERS) from rough and active site decorated surfaces. Using this as a reference point ( the flat single crystal with no active sites) we were, for the first time, able to follow the changes in surface chemistry which are induced by the ubiquitous oxidation reduction cycle (ORC) which is usually employed to create SERS. Our results directly demonstrate that with only 2 layers of $\mathrm{Ag}$ restructured in the ORC, that the surface becomes more acidic chemically. That is, it becomes more electron deficient. This occurs on sites which develop as a result of the roughening. Associated with this chemistry change is also a general increase in the overall intensity of the Raman scattering. The conclusion, which must be viewed as a caution for all investigators using SERS, is that a rough surface presents active sites which contain molecules that will dominate the signal--and that these sites are different chemically from those found of flat single crystals of the same material.

Quartz Crystal Microbalance. Bibliography Items [3 and 9]

As part of our support plan we decided two years ago to construct a facility in which direct mass changes on an electrode could be measured, independent of calibration by another technique. To that end we obtained plans from Prof. Dan Buttry at the University of Wyoming, who is an expert in the development of vibrating quartz crystal substrates as electrodes in electrochemical cells. In the published work we report studies of $\mathrm{Cd}, \mathrm{Tl}$ and $\mathrm{Pb}$ adsorbed at underpotential on polycrystalline $\mathrm{Ag}$. The quality of the data are exceptional. We have sufficient sensitivity to easily observe $3 \%$ of a monolayer. This amounts to a mass sensitivity of less than one nanogram! The $\mathrm{Pb}$ and $\mathrm{Tl}$ experiments were designed to test the device with fairly massive adsorbates. Cd was chosen because of our previous experience studying Cd monolayers with nonlinear optics.

We discovered that $\mathrm{Cd}$ and $\mathrm{Pb}$, for the most part, form deposits which show one-toone relationships between the expected amount of charge neutralization and the amount of mass in the layer. There are some subtleties associated with double layer reorganization which have yet to analyze. These appear as small peaks in the current that are not correlated with sudden changes in mass. This is an interesting effect that we are looking forward to studying further. The biggest surprise had to do with the behavior of Tl. This metal forms two well-defined monolayers prior to bulk plating. While the 
second monolayer charge flow corresponds to the mass change as expected, the first monolayer is associated with nearly double the necessary neutralization charge. This is the first superequivalent charge flow that had been identified using the QCM. In the past, electrochemists have attributed this large charge flow with a surface roughness factor greater than unity. Now we know that $\mathrm{Tl}$ must reorganize the double layer in a substantial way. Co-adsorption of a positive ion would also explain this effect. However, this would only be possible for the first layer, an interpretation which we feel is unlikely.

\section{Summary of Progress on Other Projects}

\section{Second Harmonic Reflectance on Ag(100)}

We have learned a great deal about the nonlinear optical properties, as influenced by the interfacial potential, on $\mathrm{Ag}(111)$. This has been in conjunction with our plan. Although we had not originally anticipated the need, we have found it necessary to spend a significant amount of time on $\mathrm{Ag}(100)$. The impact of these experiments comes from the fact that the surface symmetry of $\mathrm{Ag}(100)$ includes the inversion operation parallel to the surface. This means that, as is true for the bulk of a centrosymmetric material, second harmonic susceptibilities whose components are in the direction of the inversion must be zero--in the electric dipole limit. Contributions from the bulk are still possible due to much weaker higher order source terms (magnetic dipole and electric quadrupole) when integrated over the penetration depth of the optical radiation. These can be independently studied on $\mathrm{Ag}(100)$ without the relatively strong competition from the dipole allowed surface terms. By measuring the anisotropic contribution to the second harmonic reflectance as functions of the applied potential and the incident photon energy, we have been able to map some remarkable characteristics of these phenomena.

Early experiments on this surface produced the expected result predicted by the phenomenological models. That is, we found an isotropic SH reflectance on a neutral surface $(Q=0)$ independent of the incident photon energy, provided the fundamental and harmonic radiation were both $\mathrm{p}$ polarized. In the p-to-s polarized configuration, we detected anisotropy which was associated with the bulk source terms described in the previous paragraph. This anisotropy was approximately an order of magnitude smaller than that seen on $\mathrm{Ag}(111)$. Since we expect the bulk contribution to be the same on the two faces, we concluded from this that it was possible to ignore the bulk anisotropy on $\operatorname{Ag}(111)$.

Upon closer examination we discovered an anomalous anisotropic behavior on $\mathrm{Ag}(100)$. These effects became apparent on a surface with a positive charge, with s-to-p polarization, and for harmonic energies above the interband threshold in Ag. This is demonstrated in the top frame of the accompanying figure, where the dotted curve was taken on a neutral surface at a harmonic energy of $4.06 \mathrm{eV}$ and the solid curve was 
measured under positive surface charge. In addition, anisotropy was observed below the interband threshold, as can be seen in the bottom frame, measured at a harmonic energy of $3.49 \mathrm{eV}$. These results are quite unexpected, since the bulk susceptibilities should not

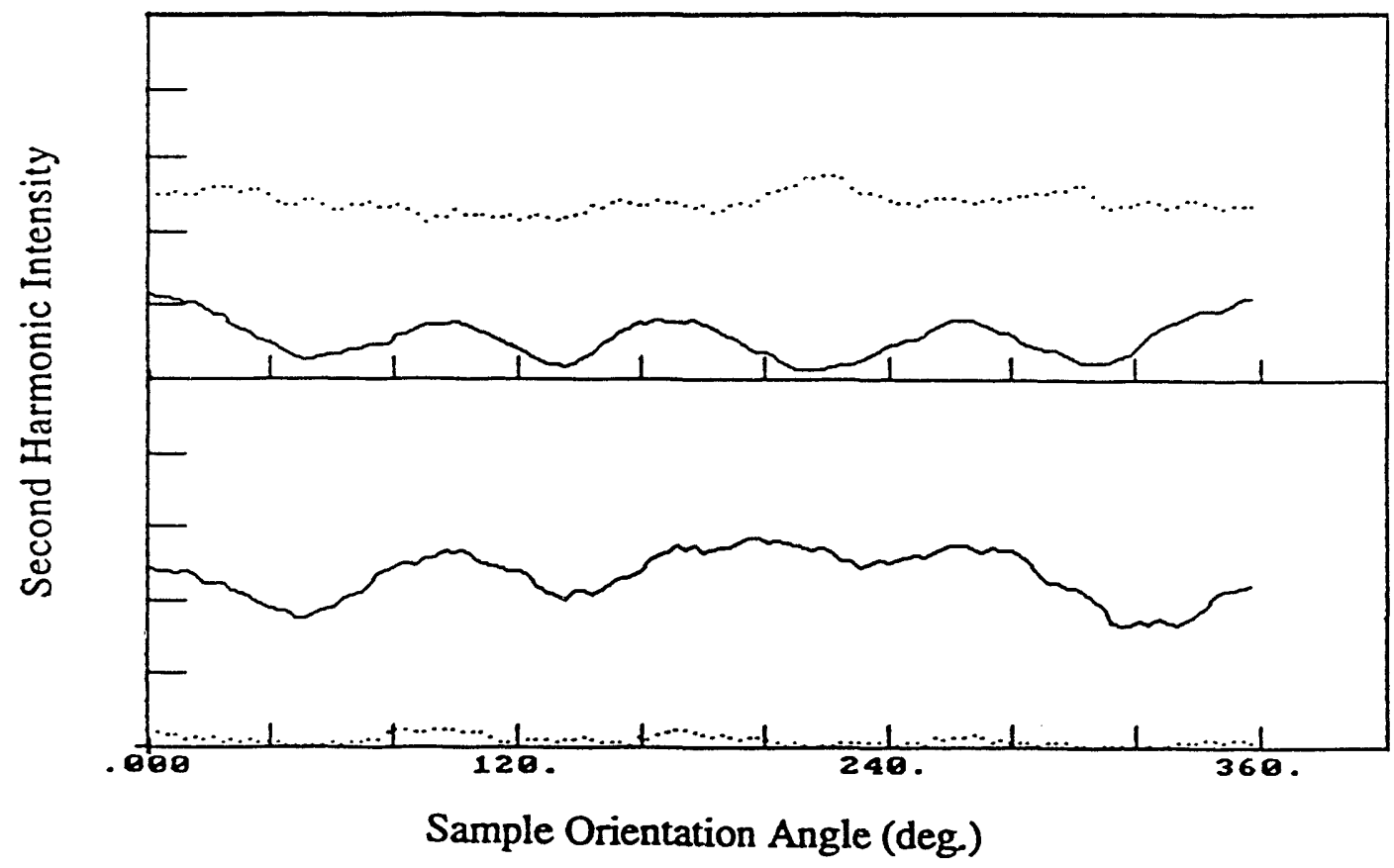

be potential dependent, and the surface susceptibilities that might lead to anisotropy in the dipole approximation should be zero. We have spent some time examining the theory of surface nonlinear optics to try to understand this anomaly. This has required a new development beyond that available in the literature. We found that the third order susceptibilities in the surface region could contribute to an anisotropic signal. Normally these are so small as to be negligible. However, on $\operatorname{Ag}(100)$ where the lower order response is symmetry forbidden, this new effect can be studied. The surface perturbation provided by the interfacial field is an ideal way of emphasizing the true interfacial contribution. Detailed analyses of these results are now in progress. However, we can say that, above the interband threshold we must be seeing an interaction between the third order contribution coming from the surface and the bulk resonant contribution. At lower energies it is quite likely that the third order contribution itself is resonant. The latter could be driven by a potential dependent surface state, which is expected on this face.

\section{Multichannel Photon Counting Surface Raman Spectrometer}

We have continued to improve our surface Raman facility, emphasizing the new detection sensitivity based upon our multichannel photomultiplier. This detector, purchased a year ago with DOE funds, is a state-of-the-art device in which weak signals are converted, one photon at a time, to a spatially resolved image on a resistive anode 
element. The overall quantum efficiency is close to $20 \%$. We are able to achieve this relatively high value by using a He-Cd laser, which puts out an excitation line at $442 \mathrm{~nm}$. This new laser was obtained during the last year through partial support (50\%) from our DOE program. The balance of the $\$ 13,000$ was provided by a grant used to support Raman spectroscopy in general Materials Sciences applications. The spectrum is dispersed with a single grating spectrometer with a high throughput. We use a special interference filter in front of the monochromator to limit the amount of elastically scattered light which can reach the detector. The effective dark count rating for this system is of the order of $10^{-4}$ counts per channel per second. This makes it possible to accumulate spectra over a relatively long period to improve the statistics. We use a multichannel analyzer to monitor the pulse height distribution coming from the detector interface. This is proportional to the position distribution of the light on the detector cathode, which in turn is proportional to wavelength. In this way we record the entire spectrum, one photon at a time, without ever rotating the grating.

As a measure of our preliminary performance we present the spectrum below, taken on $\mathrm{Si}(100)$ in air using $80 \mathrm{~mW}$ of incident power, an entrance slit width of $0.1 \mathrm{~mm}$, and an integration time of $60 \mathrm{~min}$. These data were taken at the third order region of the phonon spectrum of Si. Third order scattering is very weak and difficult to observe under any conditions. The most unusual feature of these data is the absence of a peak at $1350 \mathrm{~cm}^{-1}$. This peak is expected when the excitation is at $514 \mathrm{~nm}$. The fact that it is missing at $442 \mathrm{~nm}$ excitation is an indication of a resonant scattering at the lower energy.

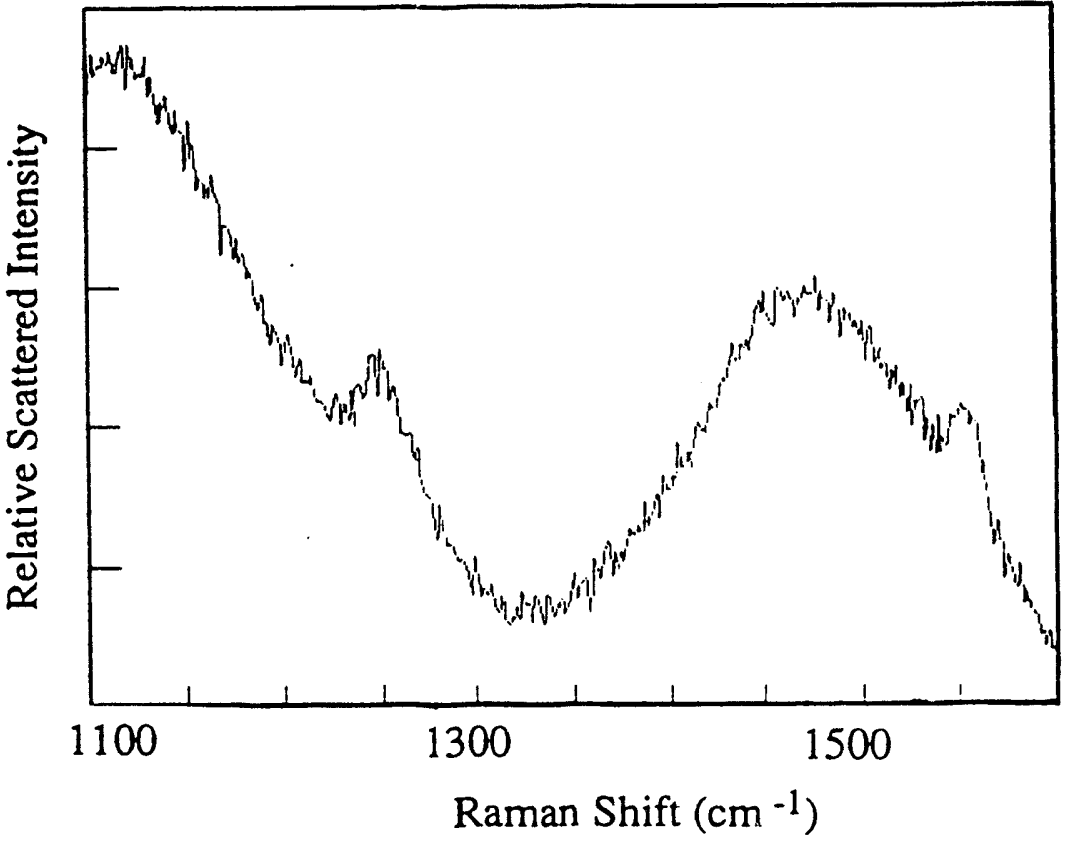

Although these results are encouraging, we know that we will have to do much better in order to achieve our goals of detecting vibrational spectra from material at the interface between the aqueous electrolyte and a flat single crystal metal electrode. We are limited by the background coming from stray light. This can be significantly reduced by better spectral purity. To accomplish this improvement we will install a series of spatial filters inside of the monochromator. This should help greatly, as it has for others who have used single monochromators for Raman spectroscopy. When the detection instrumentation is in place and optimized we will re-install our surface plasmon excitation device which we had previously operated with our more conventional 
spectrometer. The new system should push back our findamental limit, which was reached in our previous experiments, associated with intrinsic detector noise.

\section{Quartz Crystal Microbalance Measurements of $\mathrm{Cu}$ on $\mathrm{Pt}$}

Recently it has been suggested that co-adsorption of ions from the solution can significantly modify the charge state of a foreign metal. It is certainly true that anions shift the UPD equilibrium through their interaction with the deposited monolayer. This is evident from changes in the UPD shift as, for example, $\mathrm{Cl}^{-}$is added to a solution. It is not expected, however, that an ion like $\mathrm{ClO}_{4}^{-}$would influence the equilibrium to any great extent. The group at Texas A\&M, using ex situ Auger spectroscopy, has shown that, depending on the coverage of $\mathrm{Cu}$, the charge on the $\mathrm{Cu}$ atoms can range from a deficiency of electrons to an excess. This is a system which is of great interest in the interfacial science community because of its simplicity and its relationship to catalysis. We have use $\mathrm{Cu}$ on $\mathrm{Pt}$ as the prototype for our surface $\mathrm{x}$-ray absorption program. In that experiment we have shown that, at one full monolayer, $\mathrm{Cu}$ is essentially neutral, in contrast to the Texas A\&M data.

To resolve this controversy we used our quartz crystal microbalance to directly measure the accumulated mass, correlating it with the electrochemically detected charge transfer.

The results are shown here. The solid curve is the cell current, registered as the monolayer, then part of a multilayer of $\mathrm{Cu}$ is deposited on polycrystalline Pt. The negative-going peaks at about $0.3 \mathrm{~V}$ and $0.22 \mathrm{~V}$ are the two portions of the monolayer. The tail near $0.05 \mathrm{~V}$ is the onset of multilayer growth. The positive peaks show thi stripping of these deposits. The noisy curve is the synthesized result taken from

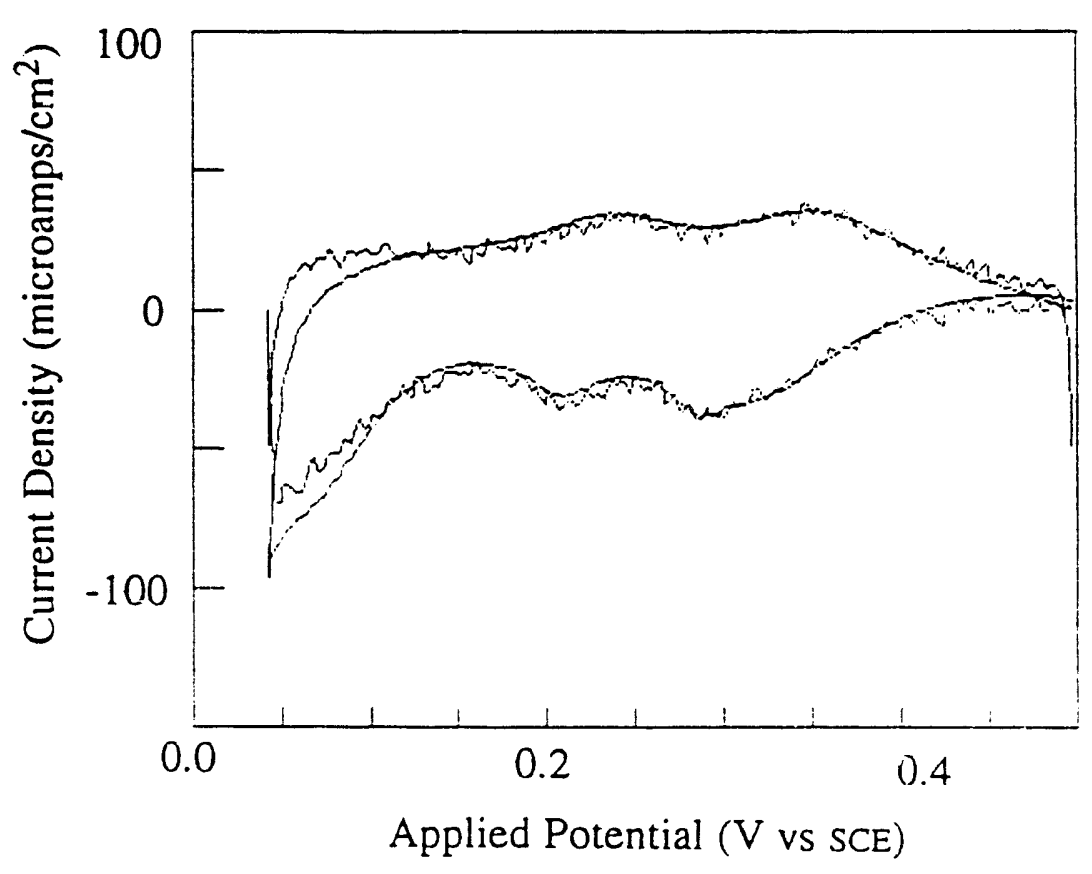
the direct mass measurement assuming that each $\mathrm{Cu}$ atom requires exactly two electrons for neutralization. We see no anomalous behavior. From these data we can say that the $\mathrm{Cu}$ discharge is exactly as expected, in cuntrast to the ex situ measurement by Auger. We see here the importance of in situ supporting experiments and the power of the mass balance in playing this role. 
In resclving the disparity between our data and those of the Texas group we should say that these results were measured in $\mathrm{SO}_{4}{ }^{2-}$, rather than $\mathrm{ClO}_{4}{ }^{-}$, and that our data were, by necessity, measured on polycrystalline $\mathrm{Pt}$, rather than on $\mathrm{Pt}(111)$.

\section{Spectroscopic Ellipsometer Development}

Part of our overall plan is to measure the various moments of the electron charge density profile at the surface of a metal electrode, to see how this is influenced by the interfacial potential. One component of this will be measurement of the first order optical susceptibility of the sample using spectroscopic ellipsometry. We have continued to build our ellipsometer, finishing the instrumentation requirements and improving those already in place.

We completed the high voltage servo control which is designed to keep the average value of the rotating analyzer signal constant. This eliminates the need for wavelength dependent normalization in the spectral portion of the experiment. It also greatly improves the short time stability of any comparison measurement. This will be needed in the electrochemical experiment. We have installed our first generation data recording hardware, based upon an HP-85 microcomputer. This allows us to signal average up to 1000 revolutions of the analyzer, transmit the data to the computer, and perform simple inversion appropriate to a two- or three-medium sample. The computer also communicates with the polarizer and the monochromator to automate calibration and spectral scanning.

We started preliminary testing of the ellipsometer and have established alignment procedures. This pointed out a problem with our original triggering circuits which instruct the data acquisition interface to take a reading. These are now being upgraded with faster optical position sensors.

We expect that this configuration will be sufficient to make preliminary measurements. However it will be essential that more versatile electronics be installed to replace the HP-85 system. We will also need to acquire a near infra-red photomultiplier to cover the entire accessible range defined by other elements of the design. This has been identified, from the outset, as part of our budget in the future.

\section{Sample Preparation}

In any surface sensitive experiment sample preparation is one of the most crucial aspects of success. In working with $\mathrm{Ag}$ we have found that some of the traditional methods have been inadequate. These include recipes provided by electrochemists in Paris which are considered by many to be the best which can be done. We have used differential capacitance to verify that our embodiment of their recipe is correct--within the ability of the capacitance to reveal differences. This is commonly accepted as the best 
way to test a clean surface. What we have found is that the optical second harmonic reflectance is a much more sensitive measure of surface quality. We must be exceptionally careful, far beyond the requirements dictated by the differential capacitance test, if we are to see the most anisotropic and largest SH response. In fact, it is only on the very best surface that we are able to observe the reconstruction which was described in the beginning of our report.

We have spent a great deal of time perfecting our surface preparation methods. To date, the best approach appears to involve chemical polishing in cyanide-based solution alternating with a hydrogen peroxide based solution. Other techniques, including electrochemical polishing and those based upon chromic acid, are far inferior to our current approach. We are continuing this investigation in an effort to improve the reproducibility of our overall program. However, we can say that, for the case of $\mathrm{Ag}$, it is likely that, without the advantage of knowing what the SH performance of the surface really is, other laboratories may be working with defective surfaces which are covered with steps, pits, and/or chemical contamination.

\section{Statement of PI and Post Doctoral Involvement}

The principal investigator, Professor Tom Furtak, devoted $30 \%$ of his time during the summer, charged to the grant. He also devoted approximately $20 \%$ of his time to this project during the academic year, of which $10 \%$ was charged to the grant from Sept. 1, 1990 to Dec. 31, 1990, and during the month of Sept. 1991.

During the entire reporting period, Dr. Yeke Tang was an important member of our group. He managed the quartz crystal microbalance experiment by himself, was an instrumental contributor to the second harmonic reflectance project, and helped complete the installation and testing of our multichannel photomultiplier. He has been a source of inspiration to us all. It was Dr. Tang's insight which led to the discovery of the surface reconstruction of $\mathrm{Ag}(111)$, one of our most significant results. He has helped guide the graduate students in our group--those working on DOE projects as well as the other business of our program.

The ultimate casualty of the reduction in budget which was imposed upon us for the second half of the period reported upon here is that we will not be able to support a postdoctoral fellow in the future. We had already promised support for Dr. Tang until the end of Sept. 1991, when we received word in Dec. 1990 that our budget was being reduced for the current renewal period. We continued his support until the end of August 1991 using DOE funds. This support of a post doctoral fellow for the time period March 1991 through August 1991 (6 months) was substituted for the second graduate student which appears in the approved budget. We had an obligation to Dr. Tang to continue his support even though funding for the position was not approved.

We are going to be significantly handicapped in achieving our proposed goals without the aid of a postdoctoral associate. Beginning in January, 1992, we will add a new 
graduate student to help with the surface Raman program. However, from now until then, that part of our research will be idle.

\section{Statement of Student Involvement}

During the grant period Lin Simpson was supported as a full-time graduate research associate (50\% during the AY, $100 \%$ during the summer). Lin is a candidate for a Ph. D. in Materials Science at CSM. He will take the Materials Science Comprehensive Examinations this November. His area of emphasis will be Solid State Physics. Lin is primarily responsible for the surface nonlinear optical spectroscopy program.

During the spring semester 1991 a senior graduate student, Li Wang, worked on the spectroscopic ellipsometer. He built and perfected the high voltage control circuit and got the entire data acquisition system running. He was supported by a full time graduate assistant grant from the Physics Department at CSM. This should be viewed as cost sharing since it directly benefited the technically approved DOE program.

We have also supported, using DOE funds, one undergraduate student during the summer, Mark Brada. He is well skilled in electronic instrumentation. His role was to improve the detection triggering on the spectroscopic ellipsometer. 
page 17

Reprints and Preprints

The attached documents represent the official product of our research. They are included as part of this Technical Progress Report and are (or will soon be) available in the open literature.

removed and

cycle

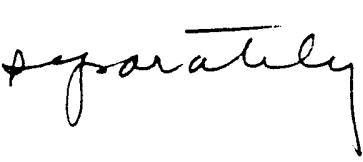



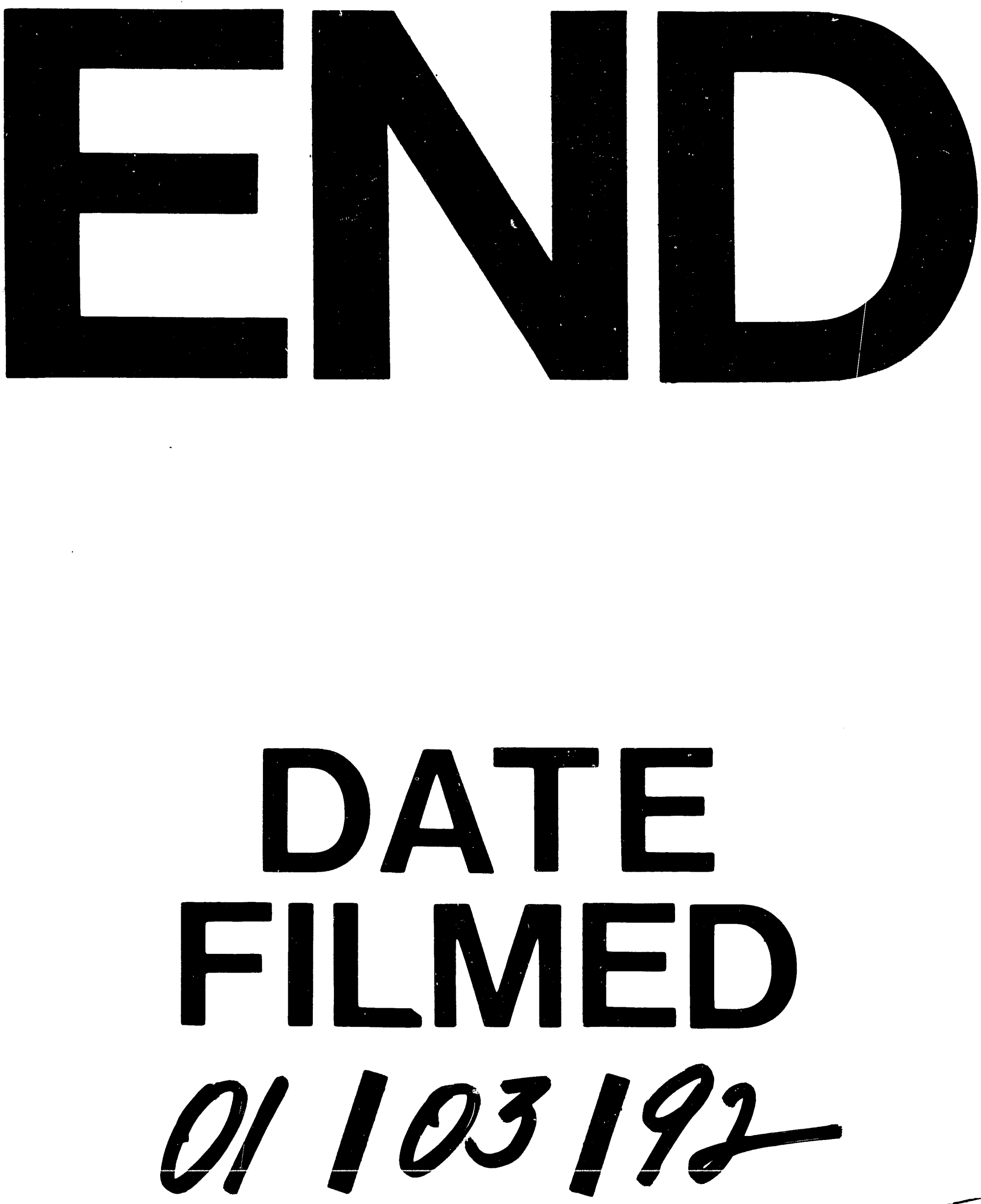
